Acta Crystallographica Section E

Structure Reports

Online

ISSN 1600-5368

\section{4-(4-Fluorobenzenesulfonamido)phenyl 4-fluorobenzenesulfonate}

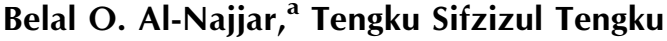 Muhammad, ${ }^{\mathbf{b}, \mathbf{c}}$ Habibah A. Wahab, ${ }^{\mathrm{a}} ¥$ Mohd Mustaqim Roslid and Hoong-Kun Fun ${ }^{d * \S}$}

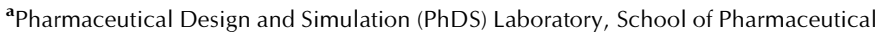
Sciences, Universiti Sains Malaysia, 11800 Minden, Pulau Pinang, Malaysia, bMalaysian Institute of Pharmaceuticals and Nutraceuticals, Ministry of Science, Technology and Innovation, SAINS@USM, No. 10, 11900 Persiaran Bukit Jambul, Pulau Pinang, Malaysia, ${ }^{\mathrm{C}}$ Department of Biological Sciences, Universiti Malaysia Terengganu, 21030 Kuala Terengganu, Terengganu, Malaysia, and ${ }^{\mathbf{d} X \text {-ray }}$ Crystallography Unit, School of Physics, Universiti Sains Malaysia, 11800 USM, Penang, Malaysia

Correspondence e-mail: hkfun@usm.my

Received 28 February 2012; accepted 6 March 2012

Key indicators: single-crystal X-ray study; $T=100 \mathrm{~K}$; mean $\sigma(\mathrm{C}-\mathrm{C})=0.002 \AA$; disorder in main residue; $R$ factor $=0.036 ; w R$ factor $=0.100$; data-to-parameter ratio $=25.5$

In the title compound, $\mathrm{C}_{18} \mathrm{H}_{13} \mathrm{~F}_{2} \mathrm{NO}_{5} \mathrm{~S}_{2}$, the complete molecule is generated by a crystallographic inversion centre, and the $\mathrm{O}$ atom and the $\mathrm{N}-\mathrm{H}$ group attached to the central ring are statistically disordered. The dihedral angle between the central and terminal benzene rings is $64.03(6)^{\circ}$. In the crystal, $\mathrm{N}-\mathrm{H} \cdots \mathrm{O}, \mathrm{C}-\mathrm{H} \cdots \mathrm{F}$ and $\mathrm{C}-\mathrm{H} \cdots \mathrm{O}$ interactions link the molecules into a three-dimensional network.

\section{Related literature}

For a related structure showing similar statistical disorder of its $\mathrm{O}$ atom and NH group, see: Al Najjar et al. (2012). For background to the biological activity of benzenesulfonates, see: Supuran et al. (2003). For the stability of the temperature controller used in the data collection, see: Cosier \& Glazer (1986).

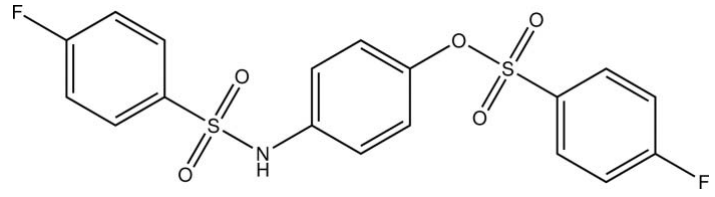

\section{Experimental}

Crystal data

$\mathrm{C}_{18} \mathrm{H}_{13} \mathrm{~F}_{2} \mathrm{NO}_{5} \mathrm{~S}_{2}$

$M_{r}=425.41$

Monoclinic, $P 2_{1} / c$

$a=8.9683$ (1) A

$b=11.0323(1) \AA$

$c=9.3314$ (1) $\AA$

$\beta=102.363(1)^{\circ}$

$V=901.85(2) \AA^{3}$

$Z=2$

Mo $K \alpha$ radiation

$\mu=0.35 \mathrm{~mm}^{-1}$

$T=100 \mathrm{~K}$

$0.37 \times 0.33 \times 0.21 \mathrm{~mm}$

\section{Data collection}

Bruker SMART APEXII CCD diffractometer

Absorption correction: multi-scan (SADABS; Bruker, 2009)

$T_{\min }=0.883, T_{\max }=0.930$

Refinement

$R\left[F^{2}>2 \sigma\left(F^{2}\right)\right]=0.036$

$w R\left(F^{2}\right)=0.100$

$S=1.09$

3235 reflections

$\mathrm{H}$-atom parameters constrained

$\Delta \rho_{\max }=0.39 \mathrm{e} \AA^{-3}$

$\Delta \rho_{\min }=-0.39 \mathrm{e}^{-3}$

Table 1

Hydrogen-bond geometry $\left(\AA{ }^{\circ}\right)$.

\begin{tabular}{llllll}
\hline$D-\mathrm{H} \cdots A$ & $D-\mathrm{H}$ & $\mathrm{H} \cdots A$ & $D \cdots A$ & \multicolumn{1}{c}{$D-\mathrm{H} \cdots A$} \\
\hline $\mathrm{N} 1-\mathrm{H} 1 N 1 \cdots \mathrm{O} 2^{\mathrm{i}}$ & 0.96 & 2.12 & $3.0630(14)$ & 169 \\
$\mathrm{C} 5-\mathrm{H} 5 A \cdots \mathrm{F} 1^{\text {ii }}$ & 0.95 & 2.37 & $3.2766(16)$ & 159 \\
$\mathrm{C} 6-\mathrm{H} 6 A \cdots \mathrm{O} 3^{\text {iii }}$ & 0.95 & 2.54 & $3.4130(17)$ & 152 & \\
$\mathrm{C} 7-\mathrm{H} 7 A \cdots \mathrm{O} 2^{\text {iv }}$ & 0.95 & 2.60 & $3.3936(17)$ & 142 & \\
\hline Symmetry codes: & (i) $-x,-y+1,-z+1 ;$ & (ii) & $-x+1, y+\frac{1}{2},-z+\frac{1}{2} ;$ & (iii) \\
$-x+1, y-\frac{1}{2},-z+\frac{1}{2} ;$ (iv) $x,-y+\frac{3}{2}, z-\frac{1}{2}$. & & & &
\end{tabular}

Data collection: APEX2 (Bruker, 2009); cell refinement: SAINT (Bruker, 2009); data reduction: $S A I N T$; $\operatorname{program}(\mathrm{s})$ used to solve structure: SHELXTL (Sheldrick, 2008); program(s) used to refine structure: SHELXTL; molecular graphics: SHELXTL; software used to prepare material for publication: $S H E L X T L$ and PLATON (Spek, 2009).

HAW gratefully acknowledges the Malaysian Ministry of Science, Technology and Innovation for the synthesis work funded by grant Nos. 311/IFN/69230112 and 304/PFARMASI/ 650545/I121. HKF thanks USM for the Research University Grant No. 1001/PFIZIK/811160.

Supplementary data and figures for this paper are available from the IUCr electronic archives (Reference: HB6664).

\title{
References
}

Al-Najjar, B. O., Tengku Muhammad, T. S., Wahab, H. A., Rosli, M. M. \& Fun, H.-K. (2012). Acta Cryst. E68, o258.

Bruker (2009). APEX2, SAINT and $S A D A B S$. Bruker AXS Inc., Madison, Wisconsin, USA.

Cosier, J. \& Glazer, A. M. (1986). J. Appl. Cryst. 19, 105-107.

Sheldrick, G. M. (2008). Acta Cryst. A64, 112-122.

Spek, A. L. (2009). Acta Cryst. D65, 148-155.

Supuran, C. T., Casini, A. \& Scozzafava, A. (2003). Med. Res. Rev. 23, 535-558.

† Additional correspondence author, e-mail: habibahw@usm.my.

§ Thomson Reuters ResearcherID: A-3561-2009. 


\title{
supporting information
}

Acta Cryst. (2012). E68, o1094 [https://doi.org/10.1107/S1600536812009877]

\section{4-(4-Fluorobenzenesulfonamido)phenyl 4-fluorobenzenesulfonate}

\author{
Belal O. Al-Najjar, Tengku Sifzizul Tengku Muhammad, Habibah A. Wahab, Mohd Mustaqim \\ Rosli and Hoong-Kun Fun
}

\section{S1. Comment}

As part of our ongoing structural investigations of benzenesulfonates (Al Najjar et al., 2012) with potential biological activities (Supuran et al., 2003), we now describe the synthesis and structure of the title compound, (I).

The asymmetric unit of the title compound consists of half the molecule with other half being generated by inversion centre. The $\mathrm{O} 1$ and $\mathrm{N} 1$ atoms occupy the same position to the central phenyl ring (Fig 1 and Fig 2), disordered with half occupancies each. A similar disordering is seen in a related structure with meta substituents on the terminal rings (Al Najjar et al., 2012), although in this case, a crystallographic twofold axis generates the complete molecule. All parameters in (I) are within normal ranges. The dihedral angle between $\mathrm{C} 1 / \mathrm{C} 6$ and $\mathrm{C} 7-\mathrm{C} 9 / \mathrm{C} 7 \mathrm{~A}-\mathrm{C} 9 \mathrm{~A}$ is $64.03(6)^{\circ}$ whereas the the $\mathrm{C} 1 / \mathrm{C} 6$ ring and its symmetry equivalent $\mathrm{C} 1 \mathrm{~A} / \mathrm{C} 6 \mathrm{~A}$ ring are constrained by symmetry to lie in a parallel orientation. In the crystal, $\mathrm{N} 1-\mathrm{H} 1 \mathrm{~N} 1 \cdots \mathrm{O} 2^{\mathrm{i}}, \mathrm{C} 5-\mathrm{H} 5 \mathrm{~A} \cdots \mathrm{F} 1^{\mathrm{ii}}, \mathrm{C} 6-\mathrm{H} 6 \mathrm{~A} \cdots \mathrm{O} 3^{\text {iii }}$ and $\mathrm{C} 7-\mathrm{H} 7 \mathrm{~A} \cdots \mathrm{O} 2^{\text {iv }}$ bonds (Table 1) link the molecules into a three-dimensional network (Fig. 3)

\section{S2. Experimental}

0.02 Mole of 4-fluorobenzenesulfonyl chloride was added to 0.01 mole of $p$-aminophenol dissolved in pyridine. Next, the reaction mixture was neutralized by adding hydrochloric acid. The precipitate formed was dissolved in $5 \%$ aqueous sodium hydroxide, and the sulfonamide recovered by adding 1:1 hydrochloric acid slowly. Re-crystallization of the product by slow evaporation of an ethyl acetate solution gave yellow blocks of (I).

\section{S3. Refinement}

$\mathrm{N}$ bound $\mathrm{H}$ atoms were located from a difference Fourier maps and refined using a riding model. The remaining $\mathrm{H}$ atoms were positioned geometrically and refined using a riding model with $\mathrm{C}-\mathrm{H}=0.95 \AA$ and $U_{\text {iso }}(\mathrm{H})=1.2 U_{\mathrm{eq}}(\mathrm{C})$. 


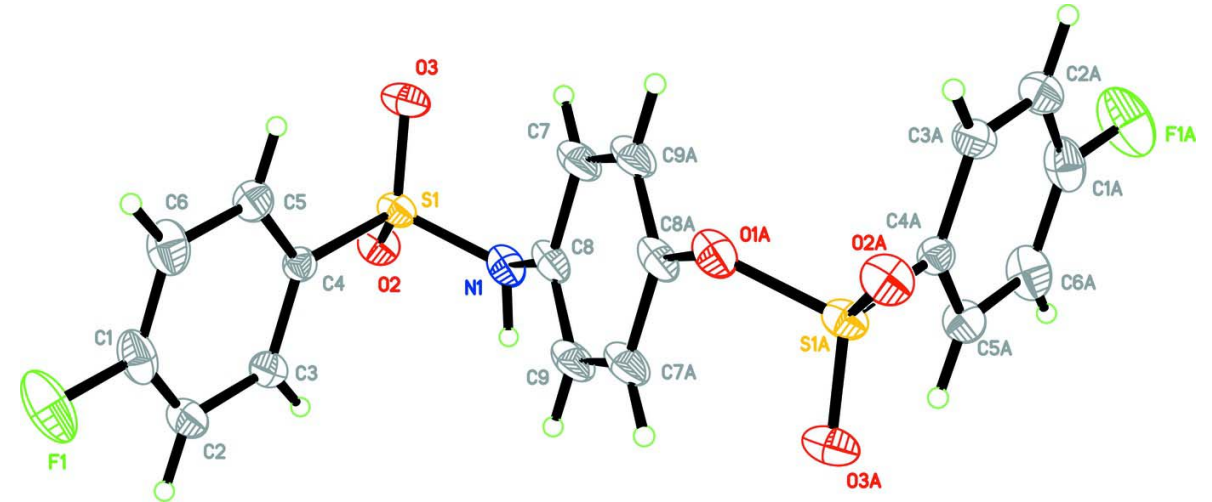

Figure 1

The first disorder component of the structure with 50\% probability displacement ellipsoids. Hydrogen atoms are shown as spheres of arbitrary radius.

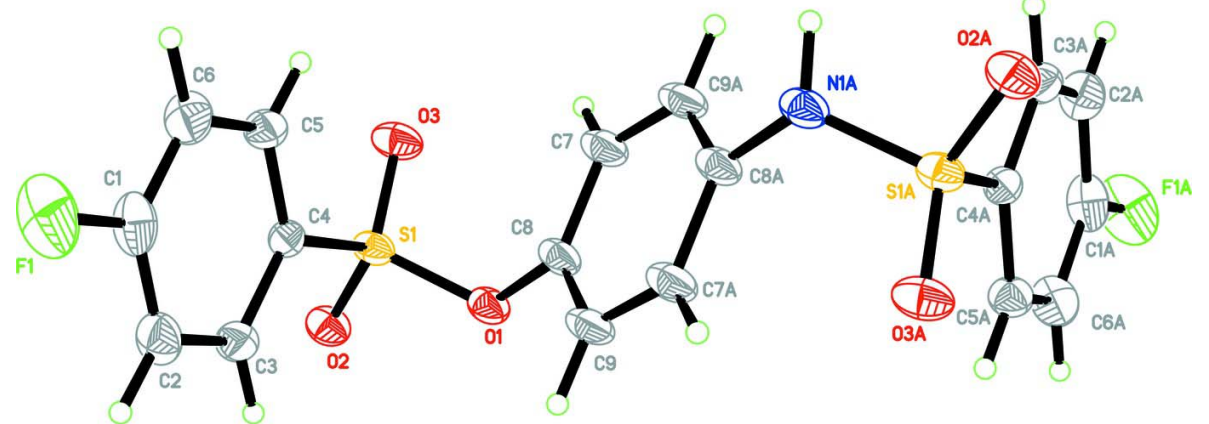

Figure 2

The second disorder component of the structure with 50\% probability displacement ellipsoids. Hydrogen atoms are shown as spheres of arbitrary radius.

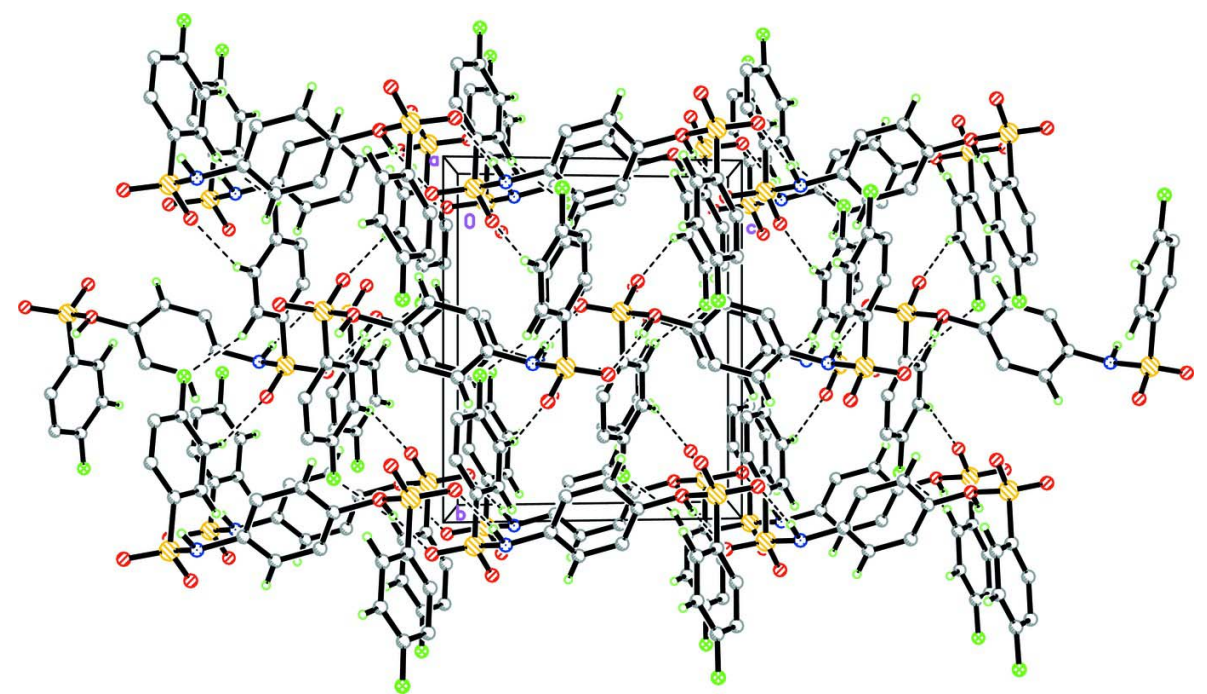

Figure 3

The crystal packing of (I). Dashed lines indicate hydrogen bonds. H atoms not involved in the hydrogen bond interactions have been omitted for clarity. 
4-(4-Fluorobenzenesulfonamido)phenyl 4-fluorobenzenesulfonate

Crystal data

$\mathrm{C}_{18} \mathrm{H}_{13} \mathrm{~F}_{2} \mathrm{NO}_{5} \mathrm{~S}_{2}$

$M_{r}=425.41$

Monoclinic, $P 2_{1} / c$

Hall symbol: -P $2 \mathrm{ybc}$

$a=8.9683$ (1) $\AA$

$b=11.0323(1) \AA$

$c=9.3314(1) \AA$

$\beta=102.363(1)^{\circ}$

$V=901.85(2) \AA^{3}$

$Z=2$

$F(000)=436$

$D_{\mathrm{x}}=1.567 \mathrm{Mg} \mathrm{m}^{-3}$

Mo $K \alpha$ radiation, $\lambda=0.71073 \AA$

Cell parameters from 7746 reflections

$\theta=2.3-32.6^{\circ}$

$\mu=0.35 \mathrm{~mm}^{-1}$

$T=100 \mathrm{~K}$

Block, yellow

$0.37 \times 0.33 \times 0.21 \mathrm{~mm}$

\section{Data collection}

\section{Bruker SMART APEXII CCD}

diffractometer

Radiation source: fine-focus sealed tube

Graphite monochromator

$\varphi$ and $\omega$ scans

Absorption correction: multi-scan

(SADABS; Bruker, 2009)

$T_{\min }=0.883, T_{\max }=0.930$

12757 measured reflections

3235 independent reflections

2940 reflections with $I>2 \sigma(I)$

$R_{\text {int }}=0.018$

$\theta_{\text {max }}=32.6^{\circ}, \theta_{\min }=2.3^{\circ}$

$h=-13 \rightarrow 13$

$k=-16 \rightarrow 16$

$l=-12 \rightarrow 14$

\section{Refinement}

Refinement on $F^{2}$

Least-squares matrix: full

$R\left[F^{2}>2 \sigma\left(F^{2}\right)\right]=0.036$

$w R\left(F^{2}\right)=0.100$

$S=1.09$

3235 reflections

127 parameters

0 restraints

Primary atom site location: structure-invariant

direct methods

Secondary atom site location: difference Fourier map

Hydrogen site location: inferred from

neighbouring sites

$\mathrm{H}$-atom parameters constrained

$w=1 /\left[\sigma^{2}\left(F_{\mathrm{o}}^{2}\right)+(0.0442 P)^{2}+0.4073 P\right]$

where $P=\left(F_{\mathrm{o}}^{2}+2 F_{\mathrm{c}}^{2}\right) / 3$

$(\Delta / \sigma)_{\max }<0.001$

$\Delta \rho_{\max }=0.39$ e $\AA^{-3}$

$\Delta \rho_{\min }=-0.39$ e $\AA^{-3}$

Special details

Experimental. The crystal was placed in the cold stream of an Oxford Cryosystems Cobra open-flow nitrogen cryostat (Cosier \& Glazer, 1986) operating at 100.0 (1) K.

Geometry. All e.s.d.'s (except the e.s.d. in the dihedral angle between two 1.s. planes) are estimated using the full covariance matrix. The cell e.s.d.'s are taken into account individually in the estimation of e.s.d.'s in distances, angles and torsion angles; correlations between e.s.d.'s in cell parameters are only used when they are defined by crystal symmetry. An approximate (isotropic) treatment of cell e.s.d.'s is used for estimating e.s.d.'s involving 1.s. planes.

Refinement. Refinement of $F^{2}$ against ALL reflections. The weighted $R$-factor $w R$ and goodness of fit $S$ are based on $F^{2}$, conventional $R$-factors $R$ are based on $F$, with $F$ set to zero for negative $F^{2}$. The threshold expression of $F^{2}>\sigma\left(F^{2}\right)$ is used only for calculating $R$-factors (gt) etc. and is not relevant to the choice of reflections for refinement. $R$-factors based on $F^{2}$ are statistically about twice as large as those based on $F$, and $R$ - factors based on ALL data will be even larger.

Fractional atomic coordinates and isotropic or equivalent isotropic displacement parameters $\left(\AA^{2}\right)$

\begin{tabular}{llllll}
\hline & $x$ & $y$ & $z$ & $U_{\text {iso }} * / U_{\text {eq }}$ & Occ. $(<1)$ \\
\hline S1 & $0.19643(3)$ & $0.57696(3)$ & $0.40553(3)$ & $0.02189(8)$ & \\
F1 & $0.48038(14)$ & $0.10387(9)$ & $0.39858(12)$ & $0.0534(3)$ &
\end{tabular}




$\begin{array}{llllll}\text { O1 } & 0.03218(11) & 0.56620(9) & 0.29564(11) & 0.0265(2) & 0.50 \\ \text { N1 } & 0.03218(11) & 0.56620(9) & 0.29564(11) & 0.0265(2) & 0.50 \\ \text { H1N1 } & -0.0391 & 0.5177 & 0.3342 & 0.032^{*} & 0.50 \\ \text { O2 } & 0.15869(11) & 0.59542(9) & 0.54583(10) & 0.02712(18) \\ \text { O3 } & 0.28500(11) & 0.66585(8) & 0.34966(11) & 0.0302(2) \\ \text { C1 } & 0.41366(16) & 0.21362(12) & 0.39786(15) & 0.0318(3) \\ \text { C2 } & 0.29275(15) & 0.22427(12) & 0.46723(16) & 0.0311(3) \\ \text { H2A } & 0.2565 & 0.1562 & 0.5119 & 0.037^{*} \\ \text { C3 } & 0.22599(13) & 0.33732(11) & 0.46964(14) & 0.0269(2) \\ \text { H3A } & 0.1428 & 0.3483 & 0.5166 & 0.032^{*} \\ \text { C4 } & 0.28240(12) & 0.43473(10) & 0.40228(12) & 0.01905(19) \\ \text { C5 } & 0.40364(13) & 0.42142(11) & 0.33233(13) & 0.0248(2) \\ \text { H5A } & 0.4402 & 0.4889 & 0.2868 & 0.030^{*} \\ \text { C6 } & 0.47055(16) & 0.30807(13) & 0.32990(15) & 0.0323(3) \\ \text { H6A } & 0.5533 & 0.2962 & 0.2826 & 0.039^{*} \\ \text { C7 } & 0.08657(15) & 0.60289(12) & 0.05220(15) & 0.0299(3) \\ \text { H7A } & 0.1450 & 0.6725 & 0.0882 & 0.036^{*} \\ \text { C8 } & 0.02046(14) & 0.53190(11) & 0.14551(13) & 0.0258(2) \\ \text { C9 } & -0.06539(15) & 0.43016(12) & 0.09444(15) & 0.0300(3) \\ \text { H9A } & -0.1097 & 0.3831 & 0.1597 & 0.036^{*}\end{array}$

Atomic displacement parameters $\left(\AA^{2}\right)$

\begin{tabular}{lllllll}
\hline & $U^{11}$ & $U^{22}$ & $U^{33}$ & $U^{12}$ & $U^{13}$ & $U^{23}$ \\
\hline S1 & $0.02541(13)$ & $0.01878(14)$ & $0.02456(14)$ & $0.00237(9)$ & $0.01219(10)$ & $0.00205(9)$ \\
F1 & $0.0779(7)$ & $0.0299(5)$ & $0.0536(6)$ & $0.0271(5)$ & $0.0170(5)$ & $0.0026(4)$ \\
O1 & $0.0272(4)$ & $0.0288(5)$ & $0.0277(4)$ & $0.0093(3)$ & $0.0154(3)$ & $0.0084(4)$ \\
N1 & $0.0272(4)$ & $0.0288(5)$ & $0.0277(4)$ & $0.0093(3)$ & $0.0154(3)$ & $0.0084(4)$ \\
O2 & $0.0327(4)$ & $0.0280(4)$ & $0.0236(4)$ & $0.0023(3)$ & $0.0127(3)$ & $-0.0018(3)$ \\
O3 & $0.0369(5)$ & $0.0206(4)$ & $0.0376(5)$ & $-0.0023(3)$ & $0.0183(4)$ & $0.0035(3)$ \\
C1 & $0.0406(6)$ & $0.0230(6)$ & $0.0289(6)$ & $0.0110(5)$ & $0.0008(5)$ & $-0.0027(5)$ \\
C2 & $0.0334(6)$ & $0.0211(5)$ & $0.0364(6)$ & $-0.0012(4)$ & $0.0020(5)$ & $0.0050(5)$ \\
C3 & $0.0237(5)$ & $0.0239(5)$ & $0.0336(6)$ & $0.0002(4)$ & $0.0073(4)$ & $0.0065(5)$ \\
C4 & $0.0185(4)$ & $0.0189(5)$ & $0.0197(4)$ & $0.0005(3)$ & $0.0042(3)$ & $-0.0003(4)$ \\
C5 & $0.0237(5)$ & $0.0267(6)$ & $0.0259(5)$ & $0.0030(4)$ & $0.0096(4)$ & $0.0008(4)$ \\
C6 & $0.0342(6)$ & $0.0342(7)$ & $0.0306(6)$ & $0.0130(5)$ & $0.0113(5)$ & $-0.0003(5)$ \\
C7 & $0.0329(6)$ & $0.0269(6)$ & $0.0349(6)$ & $0.0075(5)$ & $0.0179(5)$ & $0.0146(5)$ \\
C8 & $0.0281(5)$ & $0.0255(5)$ & $0.0281(5)$ & $0.0116(4)$ & $0.0159(4)$ & $0.0120(4)$ \\
C9 & $0.0313(6)$ & $0.0289(6)$ & $0.0351(6)$ & $0.0076(4)$ & $0.0188(5)$ & $0.0162(5)$
\end{tabular}

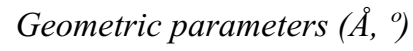

\begin{tabular}{llll}
\hline $\mathrm{S} 1-\mathrm{O} 3$ & $1.4289(9)$ & $\mathrm{C} 3-\mathrm{H} 3 \mathrm{~A}$ & 0.9500 \\
$\mathrm{~S} 1-\mathrm{O} 2$ & $1.4351(9)$ & $\mathrm{C} 4-\mathrm{C} 5$ & $1.3905(15)$ \\
$\mathrm{S} 1-\mathrm{O} 1$ & $1.6089(11)$ & $\mathrm{C} 5-\mathrm{C} 6$ & $1.3893(17)$ \\
$\mathrm{S} 1-\mathrm{C} 4$ & $1.7515(11)$ & $\mathrm{C} 5-\mathrm{H} 5 \mathrm{~A}$ & 0.9500 \\
$\mathrm{~F} 1-\mathrm{C} 1$ & $1.3499(15)$ & $\mathrm{C} 6-\mathrm{H} 6 \mathrm{~A}$ & 0.9500 \\
$\mathrm{O} 1-\mathrm{C} 8$ & $1.4332(16)$ & $\mathrm{C} 7-\mathrm{C} 9$ & $1.389(2)$
\end{tabular}




\begin{tabular}{|c|c|c|c|}
\hline $\mathrm{O} 1-\mathrm{H} 1 \mathrm{~N} 1$ & 0.9606 & $\mathrm{C} 7-\mathrm{C} 8$ & $1.3943(16)$ \\
\hline $\mathrm{C} 1-\mathrm{C} 6$ & $1.374(2)$ & $\mathrm{C} 7-\mathrm{H} 7 \mathrm{~A}$ & 0.9500 \\
\hline $\mathrm{C} 1-\mathrm{C} 2$ & $1.382(2)$ & $\mathrm{C} 8-\mathrm{C} 9$ & $1.387(2)$ \\
\hline $\mathrm{C} 2-\mathrm{C} 3$ & $1.3858(18)$ & $\mathrm{C} 9-\mathrm{C}^{\mathrm{i}}$ & $1.389(2)$ \\
\hline $\mathrm{C} 2-\mathrm{H} 2 \mathrm{~A}$ & 0.9500 & C9-H9A & 0.9500 \\
\hline $\mathrm{C} 3-\mathrm{C} 4$ & $1.3936(16)$ & & \\
\hline $\mathrm{O} 3-\mathrm{S} 1-\mathrm{O} 2$ & $119.57(6)$ & $\mathrm{C} 5-\mathrm{C} 4-\mathrm{C} 3$ & $121.74(11)$ \\
\hline $\mathrm{O} 3-\mathrm{S} 1-\mathrm{O} 1$ & $108.83(6)$ & $\mathrm{C} 5-\mathrm{C} 4-\mathrm{S} 1$ & $119.61(9)$ \\
\hline $\mathrm{O} 2-\mathrm{S} 1-\mathrm{O} 1$ & $103.25(5)$ & $\mathrm{C} 3-\mathrm{C} 4-\mathrm{S} 1$ & $118.64(8)$ \\
\hline $\mathrm{O} 3-\mathrm{S} 1-\mathrm{C} 4$ & $109.11(5)$ & $\mathrm{C} 6-\mathrm{C} 5-\mathrm{C} 4$ & $119.12(12)$ \\
\hline $\mathrm{O} 2-\mathrm{S} 1-\mathrm{C} 4$ & $109.49(5)$ & $\mathrm{C} 6-\mathrm{C} 5-\mathrm{H} 5 \mathrm{~A}$ & 120.4 \\
\hline $\mathrm{O} 1-\mathrm{S} 1-\mathrm{C} 4$ & $105.62(5)$ & $\mathrm{C} 4-\mathrm{C} 5-\mathrm{H} 5 \mathrm{~A}$ & 120.4 \\
\hline $\mathrm{C} 8-\mathrm{O} 1-\mathrm{S} 1$ & $120.53(7)$ & $\mathrm{C} 1-\mathrm{C} 6-\mathrm{C} 5$ & $118.09(12)$ \\
\hline $\mathrm{C} 8-\mathrm{O} 1-\mathrm{H} 1 \mathrm{~N} 1$ & 107.7 & $\mathrm{C} 1-\mathrm{C} 6-\mathrm{H} 6 \mathrm{~A}$ & 121.0 \\
\hline $\mathrm{S} 1-\mathrm{O} 1-\mathrm{H} 1 \mathrm{~N} 1$ & 113.2 & $\mathrm{C} 5-\mathrm{C} 6-\mathrm{H} 6 \mathrm{~A}$ & 121.0 \\
\hline $\mathrm{F} 1-\mathrm{C} 1-\mathrm{C} 6$ & $118.34(13)$ & $\mathrm{C} 9-\mathrm{C} 7-\mathrm{C} 8$ & $118.74(13)$ \\
\hline $\mathrm{F} 1-\mathrm{C} 1-\mathrm{C} 2$ & $117.73(13)$ & $\mathrm{C} 9 \mathrm{i}-\mathrm{C} 7-\mathrm{H} 7 \mathrm{~A}$ & 120.6 \\
\hline $\mathrm{C} 6-\mathrm{C} 1-\mathrm{C} 2$ & $123.93(12)$ & $\mathrm{C} 8-\mathrm{C} 7-\mathrm{H} 7 \mathrm{~A}$ & 120.6 \\
\hline $\mathrm{C} 1-\mathrm{C} 2-\mathrm{C} 3$ & $117.99(12)$ & $\mathrm{C} 9-\mathrm{C} 8-\mathrm{C} 7$ & $121.28(12)$ \\
\hline $\mathrm{C} 1-\mathrm{C} 2-\mathrm{H} 2 \mathrm{~A}$ & 121.0 & $\mathrm{C} 9-\mathrm{C} 8-\mathrm{O} 1$ & $117.95(10)$ \\
\hline $\mathrm{C} 3-\mathrm{C} 2-\mathrm{H} 2 \mathrm{~A}$ & 121.0 & $\mathrm{C} 7-\mathrm{C} 8-\mathrm{O} 1$ & $120.67(13)$ \\
\hline $\mathrm{C} 2-\mathrm{C} 3-\mathrm{C} 4$ & $119.13(11)$ & $\mathrm{C} 8-\mathrm{C} 9-\mathrm{C}^{\mathrm{i}}$ & $119.98(11)$ \\
\hline $\mathrm{C} 2-\mathrm{C} 3-\mathrm{H} 3 \mathrm{~A}$ & 120.4 & $\mathrm{C} 8-\mathrm{C} 9-\mathrm{H} 9 \mathrm{~A}$ & 120.0 \\
\hline $\mathrm{C} 4-\mathrm{C} 3-\mathrm{H} 3 \mathrm{~A}$ & 120.4 & $\mathrm{C} 77^{\mathrm{i}}-\mathrm{C} 9-\mathrm{H} 9 \mathrm{~A}$ & 120.0 \\
\hline $\mathrm{O} 3-\mathrm{S} 1-\mathrm{O} 1-\mathrm{C} 8$ & $59.87(10)$ & $\mathrm{O} 1-\mathrm{S} 1-\mathrm{C} 4-\mathrm{C} 3$ & $-71.39(10)$ \\
\hline $\mathrm{O} 2-\mathrm{S} 1-\mathrm{O} 1-\mathrm{C} 8$ & $-172.10(9)$ & $\mathrm{C} 3-\mathrm{C} 4-\mathrm{C} 5-\mathrm{C} 6$ & $-0.18(18)$ \\
\hline $\mathrm{C} 4-\mathrm{S} 1-\mathrm{O} 1-\mathrm{C} 8$ & $-57.16(10)$ & $\mathrm{S} 1-\mathrm{C} 4-\mathrm{C} 5-\mathrm{C} 6$ & $-179.74(10)$ \\
\hline $\mathrm{F} 1-\mathrm{C} 1-\mathrm{C} 2-\mathrm{C} 3$ & $178.60(12)$ & $\mathrm{F} 1-\mathrm{C} 1-\mathrm{C} 6-\mathrm{C} 5$ & $-178.59(12)$ \\
\hline $\mathrm{C} 6-\mathrm{C} 1-\mathrm{C} 2-\mathrm{C} 3$ & $-0.7(2)$ & $\mathrm{C} 2-\mathrm{C} 1-\mathrm{C} 6-\mathrm{C} 5$ & $0.7(2)$ \\
\hline $\mathrm{C} 1-\mathrm{C} 2-\mathrm{C} 3-\mathrm{C} 4$ & $0.23(19)$ & $\mathrm{C} 4-\mathrm{C} 5-\mathrm{C} 6-\mathrm{C} 1$ & $-0.25(19)$ \\
\hline $\mathrm{C} 2-\mathrm{C} 3-\mathrm{C} 4-\mathrm{C} 5$ & $0.18(18)$ & $\mathrm{C} 9-\mathrm{C} 7-\mathrm{C} 8-\mathrm{C} 9$ & $-0.15(19)$ \\
\hline $\mathrm{C} 2-\mathrm{C} 3-\mathrm{C} 4-\mathrm{S} 1$ & $179.75(10)$ & $\mathrm{C} 9-\mathrm{C} 7-\mathrm{C} 8-\mathrm{O} 1$ & $-176.54(11)$ \\
\hline $\mathrm{O} 3-\mathrm{S} 1-\mathrm{C} 4-\mathrm{C} 5$ & $-8.66(11)$ & $\mathrm{S} 1-\mathrm{O} 1-\mathrm{C} 8-\mathrm{C} 9$ & $122.24(11)$ \\
\hline $\mathrm{O} 2-\mathrm{S} 1-\mathrm{C} 4-\mathrm{C} 5$ & $-141.24(9)$ & $\mathrm{S} 1-\mathrm{O} 1-\mathrm{C} 8-\mathrm{C} 7$ & $-61.25(13)$ \\
\hline $\mathrm{O} 1-\mathrm{S} 1-\mathrm{C} 4-\mathrm{C} 5$ & $108.18(10)$ & $\mathrm{C} 7-\mathrm{C} 8-\mathrm{C} 9-\mathrm{C} 7^{\mathrm{i}}$ & $0.2(2)$ \\
\hline $\mathrm{O} 3-\mathrm{S} 1-\mathrm{C} 4-\mathrm{C} 3$ & $171.77(9)$ & $\mathrm{O} 1-\mathrm{C} 8-\mathrm{C} 9-\mathrm{C}^{\mathrm{i}}$ & $176.64(11)$ \\
\hline $\mathrm{O} 2-\mathrm{S} 1-\mathrm{C} 4-\mathrm{C} 3$ & $39.18(11)$ & & \\
\hline
\end{tabular}

Symmetry code: (i) $-x,-y+1,-z$.

Hydrogen-bond geometry $\left(A,{ }^{\circ}\right)$

\begin{tabular}{lllll}
\hline$D-\mathrm{H} \cdots A$ & $D-\mathrm{H}$ & $\mathrm{H} \cdots A$ & $D \cdots A$ & $D-\mathrm{H} \cdots A$ \\
\hline $\mathrm{N} 1-\mathrm{H} 1 N 1 \cdots \mathrm{O} 2^{\mathrm{ii}}$ & 0.96 & 2.12 & $3.0630(14)$ & 169 \\
$\mathrm{C} 5-\mathrm{H} 5 A \cdots \mathrm{F} 1^{\mathrm{iii}}$ & 0.95 & 2.37 & $3.2766(16)$ & 159
\end{tabular}


supporting information

$\begin{array}{llllr}\mathrm{C} 6-\mathrm{H} 6 A \cdots \mathrm{O} 3^{\text {iv }} & 0.95 & 2.54 & 3.4130(17) & 152 \\ \mathrm{C} 7-\mathrm{H} 7 A \cdots \mathrm{O} 2^{\mathrm{v}} & 0.95 & 2.60 & 3.3936(17) & 142\end{array}$

Symmetry codes: (ii) $-x,-y+1,-z+1$; (iii) $-x+1, y+1 / 2,-z+1 / 2$; (iv) $-x+1, y-1 / 2,-z+1 / 2$; (v) $x,-y+3 / 2, z-1 / 2$. 